\title{
Erratum: Electronic and optical properties of two-dimensional InSe from a DFT-parametrized tight-binding model [Phys. Rev. B 94, 245431 (2016)]
}

\author{
S. J. Magorrian, V. Zólyomi, and V. I. Fal'ko \\ (Received 25 July 2017; published 10 August 2017)
}

DOI: 10.1103/PhysRevB.96.079905

\section{SPIN-ORBIT COUPLING}

In the published paper we analyzed selection rules and the polarization of photons for optical interband transitions neglecting spin-orbit coupling (SOC). In the absence of SOC the electron-spin orientation is preserved in the transition between the valence and the conduction bands ( $v$ and $c$, respectively). Emission of in-plane polarized light therefore requires a difference in orbital angular momentum $\Delta L_{z}= \pm 1$ between the initial and the final states, which is impossible between $c$ and $v$ since they are nondegenerate at the $\Gamma$ point in InSe. Atomic SOC, $\mathbf{L} \cdot \mathbf{s}$, opens a spin-flip channel for the interband optical transitions, allowing angular momentum \pm 1 to be transferred to the photon by an electron spin flip. The interaction with in-plane $\sigma^{ \pm}$-polarized light can be described using $\delta H=\frac{e}{c m_{e}} \lambda_{\mathrm{SO}}\left(s^{+} \mathbf{A}^{-}+s^{-} \mathbf{A}^{+}\right)$, applied to Eq. (28), where $\mathbf{A}^{ \pm}=A(\hat{\mathbf{x}} \pm i \hat{\mathbf{y}}) / \sqrt{2}$ corresponds to $\sigma^{ \pm}$-polarized light, and $s^{+(-)}$is the spin angular momentum raising (lowering) operator.

We evaluated the coupling constant $\lambda_{\text {SO }}^{c v} \simeq 0.55 \hbar / \AA$ in the monolayer for the $c \rightarrow v$ transition across the principal band gap by examining the changes in the DFT bands at $\Gamma$ induced by SOC due to the admixture of $p_{x, y}$ orbitals to the $p_{z}$-dominated valence band. $\lambda_{S O}^{c v}$ has a nonzero value due to the opposite symmetry of $c$-and $v$-band states under $\sigma_{h}$ reflection in the monolayer, so the coupling $\delta H$ is invariant with respect to the symmetry group $D_{3 h}$ of the crystal. In the stoichiometric monolayer $\mathrm{In}_{2} \mathrm{Se}_{2}$ this gives an absorption coefficient of $2.8 \%$ for in-plane polarized light incident perpendicular to the two-dimensional crystal. In few-layer InSe the effect of SOC on the band-edge states is weaker than in the monolayer, so $\lambda_{\text {SO }}^{c v}$ and the absorption coefficient for the spin-flip transition will be smaller than in the monolayer.

\section{CORRECTION TO EQ. (28)}

The off-diagonal matrix elements between $H_{c}$ and $H_{v_{1}}$ in Eq. (28) arising from the coupling of the transition between these bands to in-plane polarized light should read $\frac{\beta_{N} e}{c m_{e}} A$ since $\beta_{N}$ has units of $\hbar / \AA$, in contrast to the matrix element between $c$ and $v$ where we use the linearity in $\mathbf{k}$ to express the matrix element with $\alpha_{N}$ as a dimensionless constant.

\section{CORRECTION TO EQ. (31)}

The effective masses given in Eq. (31) are hole effective masses and are therefore $m=-0.31$ and $m^{\prime}=-0.45$ in band $v_{1}$ and $m=-0.30$ and $m^{\prime}=-0.45$ in band $v_{2}$ in units of $m_{e}$.

\section{CORRECTIONS TO APPENDIX A}

In Appendix A Sec. $2 \boldsymbol{b}$ (M-X off-diagonal elements) the matrix element $H_{M_{p y}, X_{p y}}^{( \pm)}$should be

$$
H_{M_{p y}, X_{p y}}^{( \pm)}=f_{1}\left(T_{\pi}^{(1)} \pm T_{\pi}^{\prime(2)}\right)-\frac{f_{5} a^{2}}{12}\left[\frac{T_{\pi}^{(1)}+T_{\sigma}^{(1)}}{L_{1}^{2}} \pm \frac{T_{\pi}^{\prime(2)}+T_{\sigma}^{\prime(2)}}{L_{2}^{2}}\right]+f_{12} T_{\pi}^{(3)}-\left[\frac{f_{16} a^{2}}{3 L_{4}^{2}}\left(T_{\pi}^{(3)}+T_{\sigma}^{(3)}\right)\right]
$$

(note the division by 3 in the final term). In the first six equations of Appendix A Sec. $2 c$ ( $M$ - $M, X$ - $X$ off-diagonal elements) $T_{s p}^{(3)}$ should be replaced by $T_{s p}^{\prime(3)}, T_{\pi}^{(3)}$ by $T_{\pi}^{\prime(3)}$, and $T_{\sigma}^{(3)}$ by $T_{\sigma}^{\prime(3)} \cdot H_{M_{p x}, M_{p y}}^{( \pm)}$should be corrected further to read

$$
H_{M_{p x}, M_{p y}}^{( \pm)}=-\frac{f_{9} \sqrt{3}}{4}\left[T_{\pi}^{(2 M)}+T_{\sigma}^{(2 M)} \pm \frac{a^{2}}{L_{3}^{2}}\left(T_{\pi}^{\prime(3)}+T_{\sigma}^{\prime(3)}\right)\right]
$$

and $H_{M_{p y}, M_{p z}}^{( \pm)}$should read

$$
H_{M_{p y}, M_{p z}}^{( \pm)}=\mp \frac{f_{11} \sqrt{3} d_{M M} a}{2 L_{3}^{2}}\left[T_{\pi}^{\prime(3)}+T_{\sigma}^{\prime(3)}\right]
$$

\section{ACKNOWLEDGMENTS}

We thank Dr. X. Chen for pointing out the need for the correction to the effective masses. We thank Dr. F. Liu and Prof. Y.-H. Wang for pointing out the need for the corrections to Appendix A. 